A Scaled Underwater Launch System Accomplished by Stress Wave Propagation Technique

This article has been downloaded from IOPscience. Please scroll down to see the full text article.

2011 Chinese Phys. Lett. 28024601

(http://iopscience.iop.org/0256-307X/28/2/024601)

View the table of contents for this issue, or go to the journal homepage for more

Download details:

IP Address: 159.226.231.78

The article was downloaded on 22/03/2012 at 05:53

Please note that terms and conditions apply. 


\title{
A Scaled Underwater Launch System Accomplished by Stress Wave Propagation Technique
}

\author{
WEI Yan-Peng(魏延鹏) $)^{1 * *}$, WANG Yi-Wei(王一伟 $)^{1}$, FANG Xin(方新 $)^{2}$, HUANG Chen-Guang(黄晨光 $)^{1}$, \\ DUAN Zhu-Ping(段祝平) $)^{2}$ \\ ${ }^{1}$ Key Laboratory of Hydrodynamics and Ocean Engineering, Institute of Mechanics, Chinese Academy of Sciences, \\ Beijing 100190 \\ ${ }^{2}$ The State Key Laboratory of Nonlinear Mechanics, Institute of Mechanics, Chinese Academy of Sciences, \\ Beijing 100190
}

(Received and accepted 3 September 2010 by WEI Yue-Guang)

\begin{abstract}
A scaled underwater launch system based on the stress wave theory and the slip Hopkinson pressure bar (SHPB) technique is developed to study the phenomenon of cavitations and other hydrodynamic features of high-speed submerged bodies. The present system can achieve a transient acceleration in the water instead of long-time acceleration outside the water. The projectile can obtain a maximum speed of $30 \mathrm{~m} / \mathrm{s}$ in about $200 \mu \mathrm{s}$ by the SHPB launcher. The cavitation characteristics in the stage of acceleration and deceleration are captured by the high-speed camera. The processes of cavitation inception, development and collapse are also simulated with the business software FLUENT, and the results are in good agreement with experiment. There is about 20-30\% energy loss during the launching processes, the mechanism of energy loss is also preliminary investigated by measuring the energy of the incident bar and the projectile.
\end{abstract}

PACS: 46.40.Cd, 47.55.Dp

Cavitations generally occur if the surface pressure of the high-speed submerged body drops below the saturated vapor pressure and the resulting negative pressures are relieved by forming gas filled and gasvapor filled cavities. ${ }^{[1]}$ Cavitations can be observed in a wide variety of propulsion and power systems like pumps, marine propellers, hydrofoils and other underwater bodies. ${ }^{[2]}$ Hydrodynamic loads of body surfaces have complex forms because of the unsteady evolution of cavitating flow, which is widely researched in theoretical and experimental aspects. ${ }^{[3-5]}$

There are two main ways to realize the cavitations experiments, one is locating the static projectile at high flow liquid such as water tunnel, ${ }^{[6,7]}$ and the other is driving the high-speed projectile into liquid. ${ }^{[8]}$ For the second way, pneumatic launching is a main method to accelerate the projectile in both scaled model and full-size model, as a result, high-pressure gases ejecting from the nozzle into the water forms a previous disturbance which seriously affects the generation of cavitations in the initial stage. ${ }^{[9]}$

Stress wave propagation technique and slip Hopkinson pressure bar (SHPB) ${ }^{[10-11]}$ are widely applied to determine the dynamic mechanics properties of materials. In the present study, a scaled underwater launch system transformed from SHPB is set up, which can effectively avoid the pneumatic disturbance during launching. This system realizes transient acceleration of the projectile in water tank, which can reach $30 \mathrm{~m} / \mathrm{s}$ in less than $200 \mu \mathrm{s}$. The process of cavitations generation, development and collapse is phenomenological description by the use of a high-speed camera. The energy loss mechanism is also preliminary studied with the help of strain test method.
As shown in Fig. 1, the scaled underwater launch system mainly consists of four parts, the launching system (1,2, 3 and 5), the water tank (4 and 10), the stain sampling system $(6,7$ and 8$)$ and the high speed camera (9). The launching system converted from SHPB is used to accelerate the incident bar. The wave signal travelling in the transmission bar and projectile can be obtained from the strain sampling system. The high speed camera is used to capture the trajectory and cavitations features. The sliding sealing structure is a very important component of the water tank, it keeps the transmission bar moving freely and seals water and air.

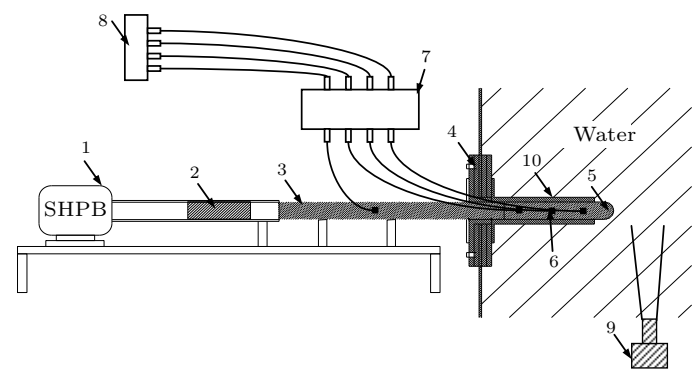

Fig. 1. The schematic of scaled underwater launch system (SULS): (1) SHPB launcher, (2) incident bar, (3) transmission bar, (4) sliding sealing, (5) projectile, (6) strain gage, (7) bridge and amplifier, (8) data sampling system, (9) high speed camera, (10) launch support.

One-dimensional stress wave theory is employed here to analysis the process of the energy transmission. The main assumptions and limitations for the system are as follows: (a) The materials of each bar is identical and mass distribution is continuous and uniform. (b) The radius of each bar is equal and $R \leq 5 L$

** To whom correspondence should be addressed. Email: osborny@msn.com

(C) 2011 Chinese Physical Society and IOP Publishing Ltd 
to fit the one-dimensional stress wave theory. (c) The velocity of incident bar has a limitation of $v$ to make sure the stress wave propagates in the bar is elastic. Here $v$ is

$$
v \leq \frac{2 \sigma_{s}}{\rho_{0} c_{0}},
$$

with $\sigma_{s}, \rho_{0}$ and $c_{0}$ being the dynamic elastic limit, density, and wave velocity of the bars.

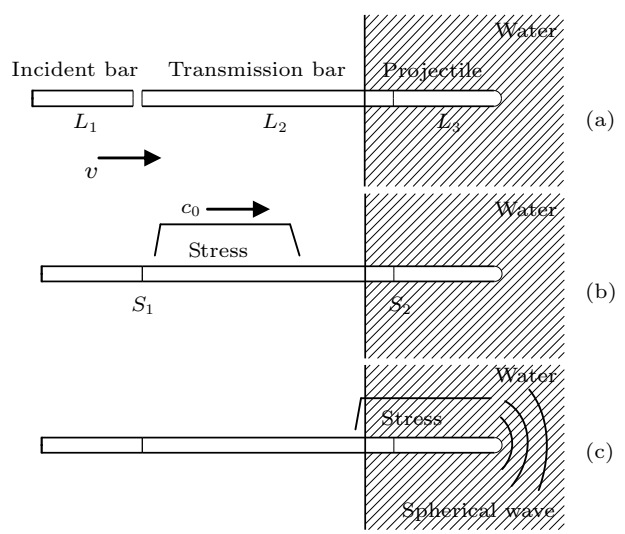

Fig. 2. Launch process schematic diagram: (a) before striking, (b) stress wave generation in transmission bar, (c) stress wave propagation into projectile.

A compressive longitudinal wave is generated once the incident bar strikes the transmission bar as shown in Fig. 2, the wave propagates along the bar as the elastic speed

$$
c_{0}=\sqrt{\frac{E}{\rho}},
$$

width of the wave is

$$
\lambda=\frac{2 L_{1}}{c_{0}}
$$

amplitude of the wave is

$$
\sigma_{e}=\frac{\rho_{0} c_{0} v}{2}
$$

where $E$ and $\rho$ are Young's modulus and density of the bar, $L_{1}$ is length of the incident bar.

All the kinetic energy of the incident bar $E_{i}$ transfers into the transmission bar in the form of compressive wave and it is separated into two parts, one is elastic energy $E_{e}$ and the other is particle kinetic energy $E_{k}$,

$$
\begin{gathered}
E_{i}=E_{e}+E_{k}, \\
E_{e}=\iint_{V} \sigma d \varepsilon d v, \\
E_{k}=\int_{M} \frac{1}{2} m v_{m}^{2} d m,
\end{gathered}
$$

where $V$ and $M$ are the volume and mass of the region that is disturbed by the wave.

If $L_{3}>L_{1}$, the compressive wave transfers into the projectile with no obstruction as $S_{2}$ is a contact surface which can endure compression force but no tensile force. Once the compressive wave reaches the projectile and water interface, a part of it is reflected back into projectile, whereas the other part is transmitted into the water as the form of spherical wave that takes away some energy. The projectile separates from the transmission bar until the reflected tensile wave reaches $S_{2}$. After several times of reflections and transmissions, the wave is dissipated and finally vanished because of lateral inertia effect, and the elastic energy transform into the kinetic energy, the projectile obtains a maximum body speed of $v_{p}$. Kinetic energy $E_{p}$ of projectile is

$$
E_{p}=\frac{1}{2} m_{p} v_{p}^{2}
$$

where $m_{p}$ is the projectile mass. The energy losses during launching $E_{l}$ and energy transfer coefficient $\eta$ are

$$
\begin{gathered}
E_{l}=E_{i}-E_{p}, \\
\eta=\frac{E_{p}}{E_{i}} .
\end{gathered}
$$

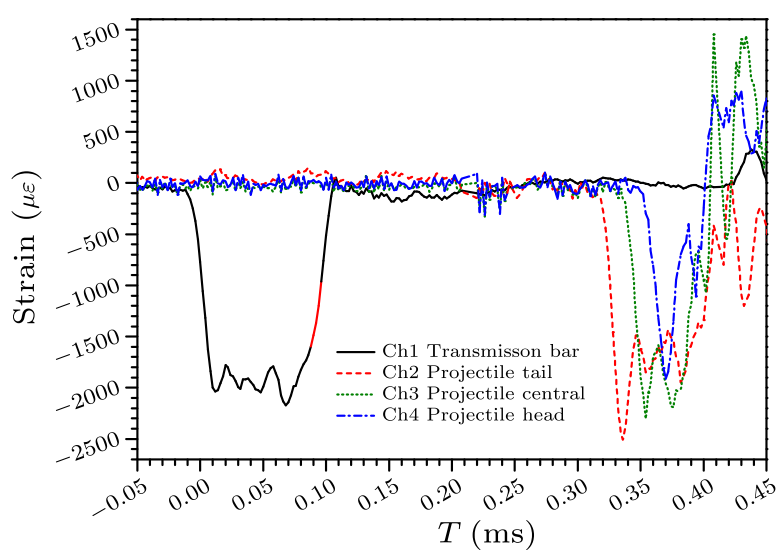

Fig. 3. Typical strain curve during launching.

As shown in Fig. 3, ch2, ch3 and ch4 are the stain perturbation which are the superposition of compressive wave and reflected tensile wave. $E_{i}$ can be derived from the strain perturbation of the transmission bar showed in ch1. $E_{e}$ and $E_{k}$ are

$$
\begin{gathered}
E_{e}=\iint_{V} \sigma d \varepsilon d v=\int_{V} \frac{1}{2} E \varepsilon^{2} d v=\frac{1}{2} E c_{0} S \int_{0}^{T} \varepsilon(t)^{2} d t, \\
E_{k}=\int_{M} \frac{1}{2} m v_{m}^{2} d m=\frac{1}{2} \rho c_{0} S \int_{0}^{T} v(t)^{2} d t,
\end{gathered}
$$

where $S$ is the cross-sectional area of the transmission bar. The conservation equation of wave front is

$$
v=c_{0} \varepsilon \text {. }
$$

The elastic modulus $E$ reads

$$
E=\rho c_{0}^{2},
$$

thus

$$
E_{k}=E_{e}
$$




$$
E_{i}=E_{e}+E_{k}=E c_{0} S \int_{0}^{T} \varepsilon(t)^{2} d t .
$$

The acceleration process of the projectile can be divided into two stages, local motion and coupled motion. At the time when the stress wave gets through $S_{2}$, the stress wave will disturb a part of particles of the projectile so that these particles will obtain identified local speed but the projectile has no body speed. This stage will last about $80 \mu \mathrm{s}$, the typical cavitations characters are the side sheet cavitations caused by lateral inertia effect, as shown in the $A$ region in Fig. 4 at $28 \mu$ s.

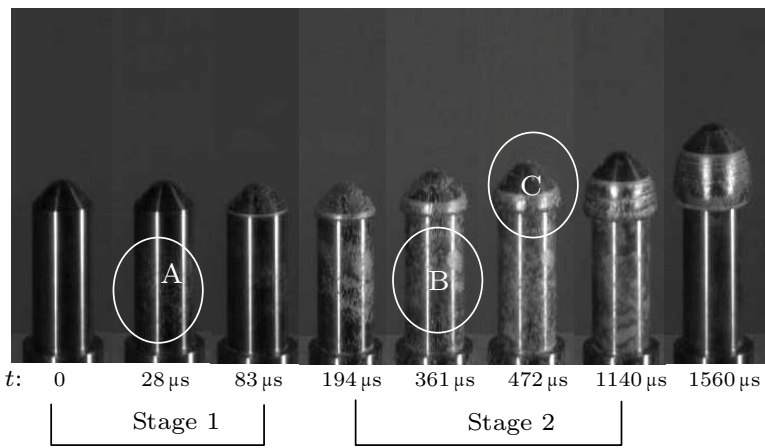

Fig. 4. The cavitations morphology captured by highspeed camera.

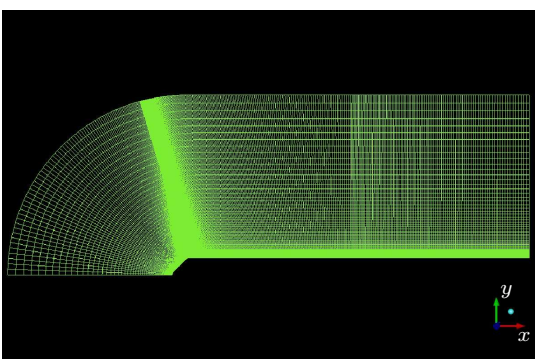

Fig. 5. The mesh of water zone.

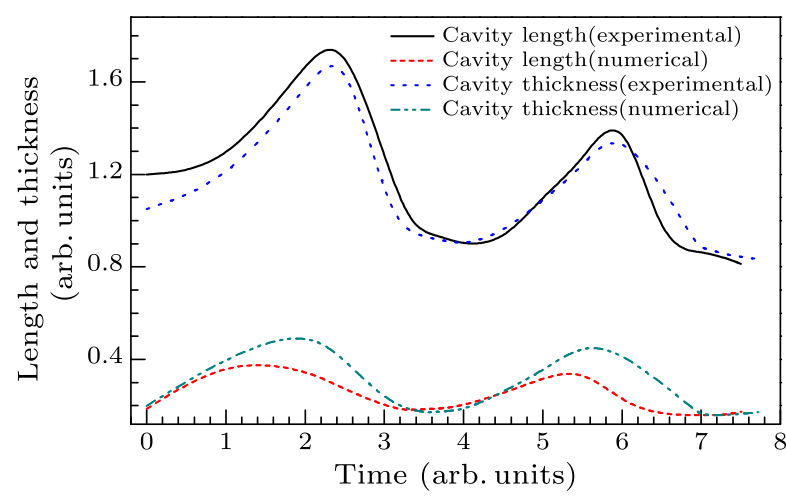

Fig. 6. Experimental and numerical results of cavity length and thickness.

In the second stage, the stress wave is dissipated because of the lateral inertial effect, elastic energy is transformed into kinetic energy gradually. This stage will last about $1000 \mu \mathrm{s}$, the typical cavitations characters are the coexistence of the side sheet cavitations as shown in the $B$ region at $361 \mu$ s in Fig. 4 and the shoulder cloud cavitations as shown in the $C$ region at $472 \mu \mathrm{s}$ in Fig. 4.

The projectile will obtain a maximum body speed after the stress wave is dissipated totally and the acceleration process is over. The projectile will decelerate by the water resistance. The typical cavitation characters of this stage are the generation, development and collapse of the shoulder cloud cavitations. A numerical simulation is carried out by FLUENT to investigate the cavitations characteristics of this stage. The two-dimensional axially symmetric model is used to depict the structure. The mesh of water zone is shown in Fig. 5.

The numerical simulation is based on the one fluid/multiple components equations, The SinghalAthavale model ${ }^{[12]}$ is employed to describe the cavitations phase transition. The background pressure is $101325 \mathrm{~Pa}$, the saturated vapor pressure is $2500 \mathrm{~Pa}$ and the initial flow velocity is $17.2685 \mathrm{~m} / \mathrm{s}$. The calculated time interval is $5 \mu \mathrm{s}$.

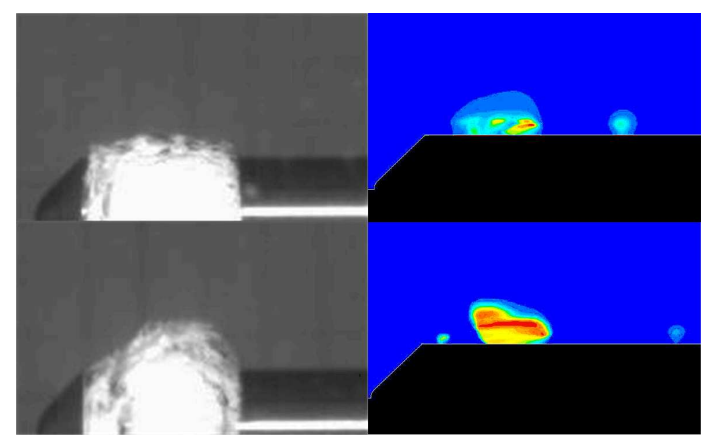

Fig. 7. Generation of cavitations after last collapse.

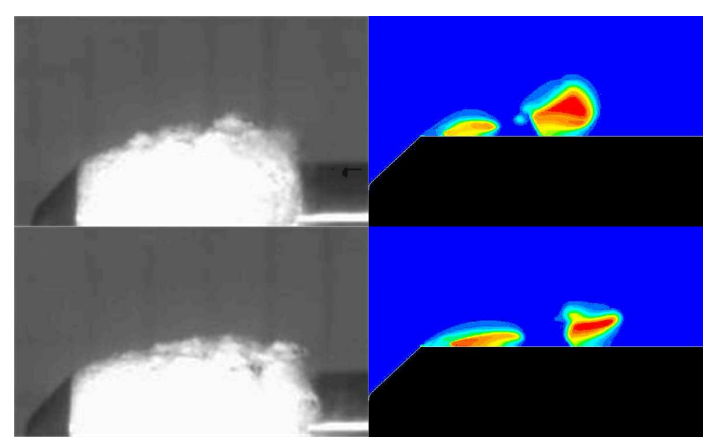

Fig. 8. Development of cavitations.

The variation of dimensionless cavity length and the cavity thickness with dimensionless time is shown in Fig. 6. The start and end position of the measured cavity length are respectively the shoulder of the projectile and the end of the shedding cavity that is not collapse yet. The development of the cavity has a cyclic trend, the maximum length is 1.89 and the maximum thickness is 0.58 . The numerical results of cavity length and cyclic trend have a good agreement with the experimental results, whereas the cavity thickness of numerical model is slightly larger than 
that of experimental. Figure 7-10 shows the numerical and experimental cavity evolution morphology at each stage.

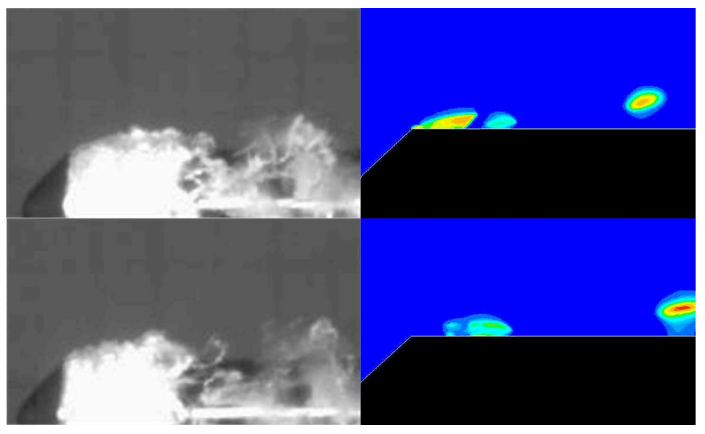

Fig. 9. Collapse of cavitations.

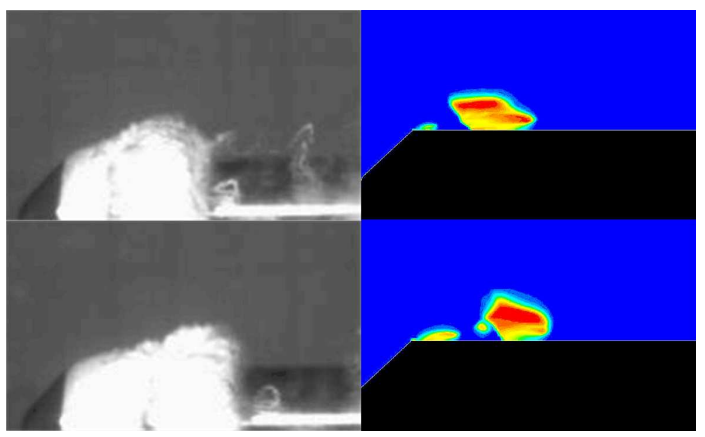

Fig. 10. New cycle of cavitations generation.

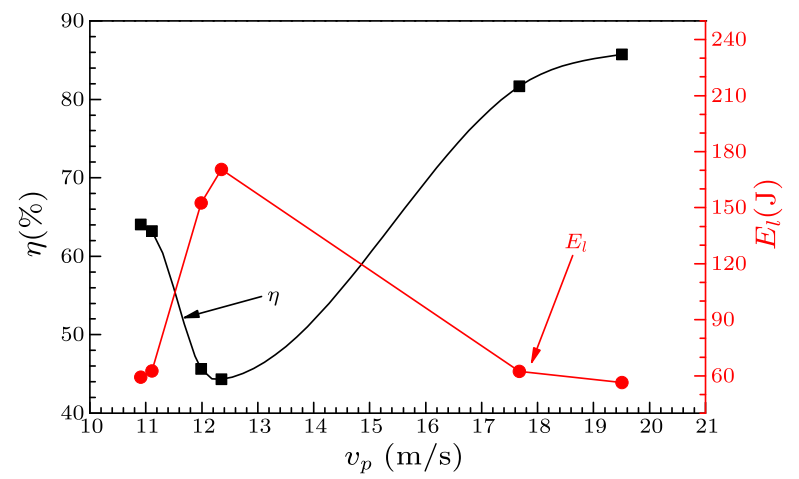

Fig. 11. Energy transfer coefficient $\eta$ and absolute energy loss $E_{J}$ at different $v_{p}$

The launch speed $v_{p}$ ranging from $10 \mathrm{~m} / \mathrm{s}$ to $30 \mathrm{~m} / \mathrm{s}$ can be obtained by adjusting the chamber pressure of SHPB. The energy efficiency coefficient $\eta$ is shown in Fig. 11. There are mainly four parts of energy loss during the launching process: (1) heat dissipation caused by friction in the contact surface of elastic bars; (2) vibration energy dissipation into water caused by lateral inertia effect; (3) transmission energy dissipation at the interface between projectile head and water; (4) assembly energy loss. As shown in Fig. 11, $\eta$ has a trend of first decrease and then increase with the increase of $v_{p}$, the maximum energy efficiency is $85.71 \%$ at maximum launch speed $19.52 \mathrm{~m} / \mathrm{s}$, and the minimum energy efficiency is only $44.32 \%$. Energy loss $E_{l}$ is below $65 \mathrm{~J}$ when $\eta$ is above $60 \%$, whereas it is more than $150 \mathrm{~J}$ when $\eta$ is around $40 \%$. The fact that $\eta$ and $E_{l}$ are volatile is mainly due to the assembly energy loss and the heat dissipation, such as the offset of bar's axis and the roughness of contact surface between bars. Assembly stability needs to improve in further work.

In summary, we have presented a scaled underwater launch system that is revised from the SHPB device, it achieves the conversion from long-time accelerating outside water to transient accelerating in water. By changing the pressure of chamber, the projectile can obtain a speed between $10 \mathrm{~m} / \mathrm{s}$ and $30 \mathrm{~m} / \mathrm{s}$ in less than $200 \mu \mathrm{s}$.

The cavitations characters in various stage of projectile motion are qualitativly researched by the highspeed camera. The evolution of cavitations includes the side sheet cavitations caused by lateral initial effect in early acceleration stage, the coexistence of side sheet cavitations and shoulder cloud cavitations in later acceleration stage and the generation, development and collapse of the shoulder cloud cavitations in deceleration stage. A numerical model is carried out by FLUENT to examine the evolution of cavitations and both the numerical and experiment results show that cavity length and thickness have a cyclic trend.

Energy efficiency during launching is also preliminarily studied. The maximum $\eta$ is $85.71 \%$ and the minimum is $44.32 \%$. The volatility of $\eta$ is mainly due to the assembly energy loss and the heat dissipation. Assembly stability needs to be improved in future work. The mechanism of vibration energy dissipation and transmission energy dissipation will be discussed elsewhere.

\section{References}

[1] Batchelor G K and Moffatt H K 2001 Appl. Mech. Rev. 54 B 89

[2] Zhang D, Liu Z P and Xiang Y P 2008 Int. J. Hydrogen. Energ. 337197

[3] Masri R and Durban D 2008 Int. J. Impact. Eng. 36830

[4] LEI X W and ZHAO X Y 2009 Chin. Phys. Lett. 26 016401

[5] Wang Y C and Chen Y W 2007 Exp. Therm. Fluid. Sci. 32403

[6] Park J T and Cutbirth J M 2003 J. Fluids Eng. 1271210

[7] Leroux J B and Billard J Y 2004 J. Fluids Eng. 12694

[8] Zhang X W and Wei Y J 2007 J. Hydrodyn. 19564

[9] Cheng Y S and Liu H 2007 J. Hydrodyn. 19403

[10] Ogawa K 2007 Int. J. Mod. Phys. B 221269

[11] Liu K X and Li X D 2006 Chin. Phys. Lett. 233045

[12] Singhal A K and Athavale M M 2001 J. Fluids Eng. 124 617 